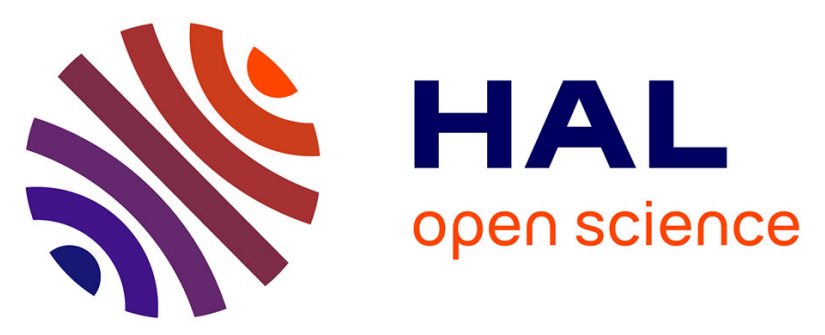

\title{
Kinase activation profile associated with TGF- $\beta$-dependent migration of HCC cells: a preclinical study
}

Emilia Fransvea, Antonio Mazzocca, Angela Santamato, Amalia Azzariti, Salvatore Antonaci, Gianluigi Giannelli

\section{To cite this version:}

Emilia Fransvea, Antonio Mazzocca, Angela Santamato, Amalia Azzariti, Salvatore Antonaci, et al.. Kinase activation profile associated with TGF- $\beta$-dependent migration of HCC cells: a preclinical study. Cancer Chemotherapy and Pharmacology, 2010, 68 (1), pp.79-86. 10.1007/s00280-010-1459-x . hal00624175

\section{HAL Id: hal-00624175 \\ https://hal.science/hal-00624175}

Submitted on 16 Sep 2011

HAL is a multi-disciplinary open access archive for the deposit and dissemination of scientific research documents, whether they are published or not. The documents may come from teaching and research institutions in France or abroad, or from public or private research centers.
L'archive ouverte pluridisciplinaire HAL, est destinée au dépôt et à la diffusion de documents scientifiques de niveau recherche, publiés ou non, émanant des établissements d'enseignement et de recherche français ou étrangers, des laboratoires publics ou privés. 
Kinase activation profile associated with TGF- $\beta$ dependent migration of HCC cells: a preclinical study

Emilia Fransvea ${ }^{\mathrm{a}}$, Antonio Mazzocca ${ }^{\mathrm{a}}$, Angela Santamato ${ }^{\mathrm{a}}$, Amalia Azzariti ${ }^{\mathrm{b}}$, Salvatore Antonaci ${ }^{\mathrm{a}}$, Gianluigi Giannelli ${ }^{\mathrm{a}}$

${ }^{\mathrm{a}}$ Department of Internal Medicine, Immunology and Infectious Diseases, Section of Internal Medicine; University of Bari Medical School, Bari, Italy, ${ }^{\mathrm{b}}$ Clinical Experimental Oncology Laboratory, National Cancer Institute Bari, Italy

Corresponding author: Prof. Gianluigi Giannelli, Dipartimento di Clinica Medica, Immunologia e Malattie Infettive, Sezione di Medicina Interna, Policlinico, Piazza G. Cesare 11, 70124 Bari, Italy. Tel: ++39 (080) 5478-127; Fax: ++39 (080) 5478-126; e-mail: g.giannelli@intmed.uniba.it 


\section{Abstract}

Purpose. To identify the molecular mechanisms responsible for tumor cell migration is essential for developing agents that can prevent the relapse or the metastatic spread of hepatocellular carcinoma (HCC). Methods. In this study, we investigated the effects of the transforming growth factor- $\beta$ receptor I inhibitor LY2109761 on two different human HCC cell lines, in vitro and in vivo. Results. LY2109761 inhibits HCC migration in a dose- dependent manner. This inhibition is associated with decreased phosphorylation of SMAD-2, FAK and $\beta 1$-integrin, and with increased levels of E-cadherin. By contrast, LY2109761 did not alter the phosphorylation pattern of p38MAPkinase. In a two- and a three-day time-course and in dose-titration experiments, LY2109761 inhibited HCC migration as well as phospho-SMAD-2 and the adhesion proteins. LY2109761 showed the best effect on day 2 at $1 \mathrm{nM}$ and for three days at $100 \mathrm{nM}$ concentration. This suggests that maximum effects were sustained for several days and were not dependent on excess concentrations. Finally, in a xenograft model of HCC, LY2109761 strongly inhibits tumor growth, intravasation and metastasis at the aforementioned lower concentrations. Conclusions. In conclusion, inhibition of transforming growth factor$\beta$ (TGF- $\beta$ ) appears to occur at low concentrations of LY2109761, that displays multiple effects on kinases that control HCC cell migration. These findings may help the design of future clinical trials with inhibitors of TGF- $\beta$. 
Key words: hepatocellular carcinoma, E-cadherin, Smad-2, tissue microenvironment, therapies 


\section{Introduction}

Hepatocellular carcinoma (HCC) is the fifth most frequent tumor, and the third cause of tumor-related death in the United States and Europe [1]. The so-called "curative" therapies, including liver transplantation, surgical resection and radiofrequency ablation, can only be offered to a minority of patients, and cancer recurrence within 3 and 5 years is a common event [2]. Unfortunately, the majority of patients are not even suitable for such "curative" treatments because of their advanced stage of disease. A significant improvement in terms of overall survival for patients with advanced disease has been achieved with sorafenib, a multi-tyrosinekinase inhibitor [3]. This finding supports the hypothesis that HCC can potentially be cured one day with appropriately targeted drugs. This approach requires, however, that the biological target is well understood and off-target effects are either minimized or re-directed to support the effect on the main signaling pathway.

The transforming growth factor (TGF)- $\beta 1$ represents an attractive target for a novel drug development strategy for HCC treatment, because of its widely reported role in chronic liver disease and in HCC, where increased levels of TGF- $\beta 1$ are associated with poor prognosis $[4,5,6]$. We have previously described the role of TGF- $\beta 1$ in triggering the epithelial mesenchymal transition in HCC [7]. As reported before, in several in vitro and in vivo models, the inhibition of the TGF- $\beta$ receptor (R) I with the selective inhibitor LY2109761 reduces HCC invasiveness, increases the expression of E-cadherin on cells, and blocks neo-vascularization via dephosphorylation of the intracellular tail of the $\beta 1$-integrin at residues T788-789 [8,9]. 
Based on these observations, we here investigated the molecular mechanisms underlying the biological activities of LY2109761. We focused on the kinase activation pattern to characterize the functional aspects of blocking TGF- $\beta$ signaling in HCC. We correlated this effect of kinase inhibition with HCC spread in vitro and in vivo. This comprehensive assessment led to some novel and unexpected findings, which suggest that TGF- $\beta$ inhibition has not only a single target hit (i.e., pSMAD2), but also down-stream of pSMAD2 multiple kinase inactivation effects. Hence, the global effect of such an inhibition may result in a more comprehensive inhibition of several important pathways involved in the pathogenesis of HCC. 


\section{Materials and Methods}

\section{Cell cultures and reagents}

Human HCC cell lines HLE and HLF, periodically purchased and used at low passages, were cultured as previously reported [10]. LY2109761 was kindly provided by Eli Lilly (Indianapolis, IN). Stock solutions were prepared at $10 \mathrm{mM}$ in dymethyl sulfoxide (DMSO) and stored in aliquots at $-20^{\circ} \mathrm{C}$. Working dilutions were made in culture medium without serum. Fibronectin ( Fn) was purchased by Calbiochem (San Diego, CA). The following antibodies were purchased as indicated: against pAkt (T308) Cell Signaling (Danvers, MA) 9275; against MEK1 (Y386) Cell Signaling 9128; against pERK 1/2 (Y202) Cell Signaling 9101, against mTOR (S2448) Cell Signaling 2971; against p-PTEN (S380) Cell Signaling 9551, against pSmad2 (S465/467) Cell Signaling 3101, against p38-MAPK (Y180/182) Cell Signaling 9211, against p-FAK (Y576/577) Cell Signaling 3281, against E-Cadherin Cell Signaling 4065, against p- $\beta 1$-integrin (T788/789) Abcam (Cambridge, MA).

\section{Migration assay}

Migration was performed as previously described [11]. Briefly, cells before migration were harvested overnight in serum-free medium. The day after the medium was changed and cells were cultured for forty-eight hours in serum-free conditions containing LY2109761 at concentrations ranging from 0.01 up $100 \mathrm{nM}$ added once or twice (every twenty-four hours). Medium was supplemented with $2 \mathrm{mmol} / \mathrm{l}$ glutamine dipeptide, glutamax, to avoid toxic catabolites (Invitrogen Carlsbad, CA), penicillin 
(100 IU/ml) and streptomycin $(100 \mu \mathrm{g} / \mathrm{ml})($ Gibco Life Technologies, Inc.). Then, cells were washed, trypsinized and seeded $\left(30 \times 10^{3}\right)$, in the absence of the drug, in the

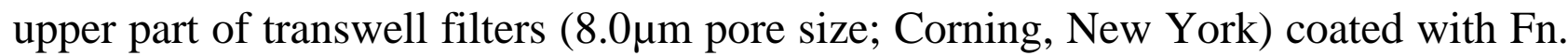
In other experiments, cells were incubated under the same experimental conditions for twenty-four hours with LY2109761 $100 \mathrm{nM}$ concentration for a time ranging from 1 up to 5 days. After incubation, cells were washed and challenged in a migration assay in the absence of the drug.

\section{HCC growth, intravasation and metastasis assay}

Tumor growth assay was performed as previously described [12]. Briefly, Fertilized white Leghorn chicken eggs (SPAFAS Inc., Norwich, CT) were kept in a forced-air incubator. On day 10, the eggs were candled, the chorioallantoic membrane (CAM) was dropped by applying gentle negative pressure, and a total of 5 $\mathrm{x} 10^{5}$ HLE cells were grafted on the dropped CAM. The eggs were then returned to a humidified $37 \mathrm{C}^{\circ}$ incubator. Tumors obtained from 19-day-old embryos were then carefully cut away from normal CAM tissue, and the wet weight of the tumor was determined. In drug experiments, different doses of LY2109761 were administered on days three and five post-inoculation of tumor cells. Measurement of intravasation and spontaneous lung metastasis using the chick embryo CAM assay was performed as previously described [12].

\section{ELISA for E-cadherin}


E-cadherin was detected in the conditioned media and cell lysates of HLE and HLF cells treated with LY2109761 at concentrations ranging from 1 up $100 \mathrm{nM}$ for two days. In time course experiments, E-cadherin was detected in the conditioned media and cell lysates of cells treated with LY2109761 at $100 \mathrm{nM}$ for three days. All the experiments were performed in serum free conditions. E-cadherin was detected in the conditioned media using quantikine ELISA kit from R\&D Systems, Inc. (Minneapolis $\mathrm{MN}$ ), and in the cell lysates using duoset IC from R\&D following manufacturer's instructions. Conditioned media were concentrated using the amicon ultra filter device from Millipore (Billerica, MA). In all the samples, protein concentrations were determined by colorimetric Bradford protein assay.

\section{Reverse phase protein microarray construction}

The reverse phase protein microarray construction was previously reported $[13,14]$. Briefly, samples were lysed, loaded into 384-well plates and printed in duplicate onto nitrocellulose-coated glass slides (FAST Slides Whatman, Florham Park, NJ) using an Aushon 2470 arrayer (Aushon BioSystems, Burlington, MA). Cellular lysate prepared from HeLa \pm Pervanadate (Becton Dickinson, Franklin Lakes, NJ) and Jurkat \pm Calyculin (Cell Signaling Technology, Danvers, MA) cell lines were printed on each array for quality control assessments. All antibodies used in this study were validated for specificity by immunoblotting prior to use on the arrays. 
Total protein values were assessed by staining the slides with Sypro Ruby Blot Stain (Molecular Probes, Eugene, OR). Stained slides were scanned with Axon GenePix (Molecular Devices, Sunnyvale, CA) and spot intensities were quantitated and normalized to total protein.

\section{Data analysis and statistical procedures}

All experiments were done at least twice. Data are presented as mean \pm SD of a representative experiment or of normalized data of pooled experiments. To compare means a repeated measure analysis of variance has been performed.

For the experiment described in Fig.1 and Fig.2, the repeated measure has been evaluated by profile of concentration. For the experiment described in Fig.5 the repeated measure by day has been compared with the baseline value (day 1). P-values lower than 0.05 have been considered significant. The analysis has been conducted by means of SAS 9.1 for PC. 


\section{Results}

We investigated the kinase profile to understand the kinase activation profile responsible for pathways involved in TGF- $\beta 1$ dependent HCC cell migration. HLE and HLF cells were incubated for forty-eight hours in serum-free medium with the TGF- $\beta$ RI kinase inhibitor LY21098761. A concentration range of 0.01 to $100 \mathrm{nM}$ was used. We also investigated whether adding the drug twice (every twenty four hours) led to an enhanced effectiveness of blocking the TGF-b pathway. In both cell lines, LY2109761 strongly inhibited the migration on Fn as indicated by a typical dose-response curve. The maximum effect was observed between 1 up to $100 \mathrm{nM}$ and adding the inhibitor a second time to the media led to a stronger inhibition $(\mathrm{p}<0.0001)$ (Fig.1A, panel A). Next, we investigated the phosphorylation of pSMAD2 and p38-MAP-Kinase in the same experiment and compared the phosphorylation pattern with the results of the migration assay. SMAD-2 was de-phosphorylated in a dose dependent manner that perfectly matches the inhibition of cell migration described in panel A (Fig 1A, panel B). By contrast, p38MAPK was not dephosphorylated even at higher concentrations (Fig.1A, panel C). Then, we investigated the adhesion molecules commonly involved in cell migration. The kinase profile analyses revealed that FAK and $\beta 1$-integrin were de-phosphorylated while Ecadherin expression was increased, $\mathrm{p}<0.0001$ (Fig.1A, panel D-F). Importantly, while adding compound to the culture increased the inhibition of the migration, doubling the treatment did not consistently increase the de-phosphorylation of 
pSMAD-2 or the adhesion molecules. Therefore, the inhibition of the TGF- $\beta$ signaling blocks HCC cell migration via selective inhibition of the SMAD-2 pathway, leads to deactivation of the adhesion molecules FAK and $\beta 1$-integrin while increasing the expression of E-cadherin. In addition to these important kinases, we also evaluated the kinase activation of those kinases generally associated with other cancer-related signal transduction pathways. As shown in Fig.1B, pAKT/mTOR and MEK/pERK1/2 were de-phosphorylated while the oncosuppressor PTEN was activated, all with a similar dose-response profile.

Based on the above described results, we restricted the range of concentration ( 1 up to $100 \mathrm{nM}$ ) prior to challenging the cells to migrate after two and three days of drug administration. In both cell lines, different drug concentrations reached the plateau effect at $1 \mathrm{nM}$ concentration at day two. After three days, the stronger effect was gained with progressively increasing doses up to $100 \mathrm{nM}$ concentration $\mathrm{p}<$ 0.0001, Fig. 2. The p-SMAD-2 and the adhesion molecules showed a pattern of dephosphorylation or increased expression consistent with the inhibition of cell migration, Fig.2. As above descibed, also pAKT/mTOR and MEK/pERK1/2 were dephosphorylated while the oncosuppressor PTEN was activated in a time- and dosedependent manner, $\mathrm{p}<0.0001$, Fig.3. Hence, $1 \mathrm{nM}$ after two days or $100 \mathrm{nM}$ of LY2109761 after three days displayed similar effectiveness on HCC cell migration as well on the kinase profile activation.

We further studied E-cadherin because of its functional role in cell migration. Untreated HLE and HLF cells display low levels of E-cadherin in the cell lysates 
while higher levels are detected in the conditioned media. However, this situation is completely reversed after LY2109761 treatment: cellular expression of E-cadherin progressively increases, while the released or soluble E-cadherin levels decrease in the conditioned media. Similarly, E-cadherin levels after treatment with LY2109761 at $100 \mathrm{nM}$ concentrations increased in the cell lysates and decreased in the conditioned media during the time-course experiments, Fig.4.

Because of the interesting time-course pattern of the kinase activation profile, we chose to treat the cells only once with $100 \mathrm{nM}$ for the migration assay. HLE and HLF cells were incubated in serum-free condition with $100 \mathrm{nM}$ of LY2109761, from 1 to 5 days. After twenty-four hours, the inhibition of HLE migration was already evident (around 30\%), but reached the strongest effect after forty-eight hours $(\mathrm{p}<0.0001)$ of incubation (around 60\%), with a progressive declining effect after 3,4 and 5 days, Fig.6. We investigated the concomitant intracellular kinase activation profile. SMAD-2 was de-phosphorylated by LY2109761 with the maximum effect being evident after forty-eight hours, $\mathrm{p}<0.0002$, corresponding to the strongest inhibition of cell migration. Consistently with the biological effect the adhesion molecules, FAK and $\beta 1$-integrin, were de-phosphorylated while E-cadherin expression was increased in a time-dependent manner $(\mathrm{p}<0.05)$ corresponding with the de-phosphorylation of p-SMAD-2. Finally, MEK/ERK1/2 and Akt/mTOR were de-phosphorylated and PTEN was activated in a time-dependent manner Fig.5.

Based on the profile kinase inhibition, we investigated in a chick embryo xenograft model, the effect of LY2109761 added every three days in a concentration 
ranging from 10 up to $50 \mathrm{mg} / \mathrm{Kg}$ [12]. As reported in Fig. 6, LY2109761 inhibits HCC metastasis in a dose-dependent manner, and furthermore also intravasation and tumor growth. 


\section{Discussion}

This study describes, for the first time, the molecular mechanisms by which the inhibitor of the TGF- $\beta$ RI LY2109761 blocks HCC migration. Taking advantage of a wide profile of kinase examination, we identified that LY2109761 selectively acts via a SMAD-dependent mechanism at different concentrations. This is also consistent with our previous study and with a study in pancreatic cancer cells $[8,15]$. In contrast to the pancreatic cancer cells, HCC cells seem to be more sensitive to LY2109761 treatment and show a five hundred fold higher sensitivity. In addition, in vivo we found that LY2109761 decreased tumor growth, intravasation and metastatization. We used a low dosage, namely $50 \mathrm{mg} / \mathrm{Kg}$ once a day for twice a week, while Melisi et al used the same drug concentration twice a day for 5 days a week [15]. Unlike the mouse xenograft model, we used a chick embryo model and thus the difference in the animal model may explain our efficacy data on the kinetic of LY2109761. Our in vivo results can be explained by the effects of LY2109761 also on other kinases commonly involved in cancer such as MEK/ERK1-2, Akt/m-TOR, PTEN. We do not know whether these pathways are activated down-stream of SMAD-2 or whether this activation is a result of unspecific inhibition by LY2109761. Hence, we cannot rule out the possibility that LY2109761 displays some indirect effect also on Akt and mTOR downstream pathways of PI3K, as reported in the case of cell hypertrophy induced by increased glucose concentration [16]. However, given the time-course activation (Fig 4), it appears more likely that LY2109761 has a strong inhibition on pSMAD2 followed then by the inhibition of down-stream kinases. If this is the case, 
LY2109761 targeting only one receptor will trigger a number of multiple cascades, which can represent a new treatment paradigm for HCC.

Independently of these unsolved specificity issues, LY2109761 demonstrates a strong association between biological effectiveness and the inhibition of kinase profile. In our study, doubling the drug concentration administration had only a small additive inhibitory effect on the HCC migration while the inhibition of the kinases associated with the migration pathways was unchanged. Furthermore, we also demonstrate that our maximum concentration, $100 \mathrm{nM}$, is still effective up to three days. Therefore, repeated treatment in a short time did not add to the inhibition and an every three day schedule was sufficient for achieving the intended inhibition of the HCC migration. Perhaps this longer lasting inhibitory effect is consistent with the mechanism of action of LY2109761. Since this small molecule is a competitive inhibitor of adenosine-triphosphate (ATP) it may not be degraded as quickly in the HCC cells [17]. Thus, repeated drug-administration may lead to drug accumulation without additional therapeutic effect and perhaps increase the potential of undesired or even toxic effects [12]. Furthermore, we demonstrate that LY2109761 modulates the functional role of down-stream molecules involved in cell motility. For instance, we show that E-cadherin is poorly expressed in the HCC invasive cells but highly detected in the conditioned medium. LY2109761 increases the cellular expression of E-cadherin and decreases its concentration in the conditioned medium. The effect of LY2109761 on the increased expression of E-cadherin is evident up to three days, further confirming the utility of this therapeutic regimen. This finding is also of 
interest because E-cadherin can be reliably detected by ELISA in biological specimens including tissue and serum, and therefore could be used as a biomarker to assess drug effectiveness.

In conclusion, our results indicate the molecular mechanisms whereby targeting TGF- $\beta$ RI inhibits HCC migration in vitro and tumor progression in vivo. We also demonstrate the optimal drug concentration and schedule administration in an in vitro and in vivo model. Then, we demonstrate drug effectiveness on both HCC cells and molecular pathways and that E-cadherin may represent a biomarker to assess therapeutic efficacy. All these data provide fundamental indications for the use of LY2109761 in future clinical trials.

\section{Acknowledgement:}

We are grateful to Dr. Michael Lahn (Eli Lilly and Company) for critical review of the manuscript. This work was supported by the Italian Association Cancer Research (AIRC) grant to GG number 202240GNN. 


\section{References}

1. El Serag H B (2004) Hepatocellular carcinoma: recent trends in the United States. Gastroenterology 127: S27-S34

2. Llovet J M, Burroughs A, Bruix J (2003) Hepatocellular carcinoma. Lancet 362: $1907-1917$

3. Llovet J M, Ricci S, Mazzaferro V, Hilgard P, Gane E, Blanc J F, de Oliveira A C et al (2008) Sorafenib in advanced hepatocellular carcinoma. N Engl J Med 359: $378-390$

4. Bedossa P, Peltier E, Terris B, Franco D, Poynard T (1995) Transforming growth factor-beta 1 (TGF-beta 1) and TGF-beta 1 receptors in normal, cirrhotic, and neoplastic human livers. Hepatology 21: 760-766

5. Tsai J F, Chuang L Y, Jeng J E, Yang M L, Chang W Y, Hsieh M Y, Lin Z Y, Tsai J H (1997) Clinical relevance of transforming growth factor-beta 1 in the urine of patients with hepatocellular carcinoma. Medicine (Baltimore) 76: 213226

6. Abou-Shady M, Baer H U, Friess H, Berberat P, Zimmermann A, Graber H, Gold L I et al (1999) Transforming growth factor betas and their signaling receptors in human hepatocellular carcinoma. Am J Surg 177: 209-215 
7. Giannelli G, Bergamini C, Fransvea E, Sgarra C, Antonaci S (2005) Laminin5 with transforming growth factor-beta1 induces epithelial to mesenchymal transition in hepatocellular carcinoma. Gastroenterology 129: 1375-1383

8. Fransvea E, Angelotti U, Antonaci S, Giannelli G (2008) Blocking transforming growth factor-beta up-regulates E-cadherin and reduces migration and invasion of hepatocellular carcinoma cells. Hepatology 47: 1557-1566

9. Fransvea E, Mazzocca A, Antonaci S, Giannelli G (2009) Targeting transforming growth factor (TGF)-betaRI inhibits activation of beta1 integrin and blocks vascular invasion in hepatocellular carcinoma. Hepatology 49: 839850

10. Bergamini C, Sgarra C, Trerotoli P, Lupo L, Azzariti A, Antonaci S, Giannelli G (2007) Laminin-5 stimulates hepatocellular carcinoma growth through a different function of alpha6beta4 and alpha3betal integrins. Hepatology 46: 1801-1809

11. Giannelli G, Bergamini C, Fransvea E, Marinosci F, Quaranta V, Antonaci S (2001) Human Hepatocellular Carcinoma (HCC) Cells Require Both alpha3beta1 Integrin and Matrix Metalloproteinases Activity for Migration and Invasion. Lab Invest 81: 613-627

12. Mazzocca A, Fransvea E, Lavezzari G, Antonaci S, Giannelli G (2009) Inhibition of transforming growth factor beta receptor I kinase blocks 
hepatocellular carcinoma growth through neo-angiogenesis regulation. Hepatology 50: 1140-1151

13. Liotta L A, Espina V, Mehta A I, Calvert V, Rosenblatt K, Geho D, Munson P J et al (2003) Protein microarrays: meeting analytical challenges for clinical applications. Cancer Cell 3: 317-325

14. Wulfkuhle J D, Aquino J A, Calvert V S, Fishman D A, Coukos G, Liotta L A, Petricoin E F, III (2003) Signal pathway profiling of ovarian cancer from human tissue specimens using reverse-phase protein microarrays. Proteomics 3: 2085-2090

15. Melisi D, Ishiyama S, Sclabas G M, Fleming J B, Xia Q, Tortora G, Abbruzzese J L, Chiao P J (2008) LY2109761, a novel transforming growth factor beta receptor type I and type II dual inhibitor, as a therapeutic approach to suppressing pancreatic cancer metastasis. Mol Cancer Ther 7: 829-840

16. Wu L, Derynck R (2009) Essential role of TGF-beta signaling in glucoseinduced cell hypertrophy. Dev Cell 17: 35-48

17. Li H Y, McMillen W T, Heap C R, McCann D J, Yan L, Campbell R M, Mundla S R et al (2008) Optimization of a dihydropyrrolopyrazole series of transforming growth factor-beta type I receptor kinase domain inhibitors: discovery of an orally bioavailable transforming growth factor-beta receptor type I inhibitor as antitumor agent. J Med Chem 51: 2302-2306 


\section{Figure legend}

Figure 1. LY2109761 inhibits HCC cell migration via SMAD-2 in a dose dependent manner and affects intracellular pathways. In Fig1A, HLE and HLF cell migration is inhibited in a dose dependent manner. However, LY2109761 exerts stronger effects if the drug was added twice to cells (every twenty-four hours), panel A. The study of TGF- $\beta$ dependent pathways performed using the kinases assay was executed on each condition tested in the migration assay revealed that this effect is achieved through the inhibition of the Smad-2 dependent and non independent pathway, panel B-C. The inhibition of adhesion molecules p-FAK (panel D and p- $\beta 1$ integrin (panel E), together with the increased expression of E-cadherin (panel F) is dose-dependent, although doubling the drug administration is not more effective that the single treatment. In Fig.1B, single or double administration of LY2109761 on HLE and HLF yield a similar inhibition of the phosphorylation of the pathways MEK/ERK and Akt/n-TOR, also including the activation of PTEN.

Figure 2. LY2109761 effectiveness in a dose-dependent and time-course experiment. Effects of LY2109761 on HLE and HLF migration at different doses and at different times. Best results were obtained using LY2109761 after twenty-four hours at $1 \mathrm{nM}$ or after seventy-two hours at $100 \mathrm{nM}$ concentration The kinase profile inhibition of the FAK and integrin $\beta 1$ as well as the E-cadherin expression showed a similar pattern. 
Figure 3. LY2109761 effectiveness on tumoral pathways in a dosedependent and time-course experiment. LY2109761 de-phosphorylates MEK/ERK and Akt/m-TOR as well as activates PTEN in a dose and time dependent manner. The inhibition of such pathways is consistent with that of cell migration, previously shown.

Figure 4. LY2109761 modulates the expression and the release of Ecadherin. E-cadherin levels were measured by ELISA in the cell lysate and conditioned media of HLE and HLF cells. In panel A, the levels of E-cadherin decrease in the conditioned medium and increase in the cell lysate in a dose dependent manner, reaching the maximum effect at $100 \mathrm{nM}$, consistently with the inhibition of cell migration described in Fig.1. In panel B, the levels of E-cadherin were investigated in a time-course experiment, in the conditioned medium and cell lysate of HLE and HLF treated with LY2109761 at $100 \mathrm{nnM}$. The best results were obtained after two days and remained similar three days after treatment.

\section{Figure 5. LY2109761 effectiveness in a time-course experiment.} Effectiveness of LY2109761 at $100 \mathrm{nM}$ on HLE and HLF migration in 5 days timecourse experiment, panel A. Kinase profile of the molecular pathways as well as receptors effective of cell motility performed in the time-course experiment at each condition, panel $\mathrm{B}$. The inhibition of the molecular pathways matches the inhibition of cell migration. 


\section{Figure 6. Dose-dependent effect of LY2109761 treatment on tumor} growth, intravasation, and metastasis of human HCC in xenograft model. Analysis of metastatic (A), intravasation (B) and growth (C) of HCC tumors treated with different doses of LY2109761. Intravasation of tumor cells was evaluated by realtime Alu PCR of genomic DNA extracted at day 7 after injection of cells. HCC dissemination to the lung was instead evaluated by fluorescent microscopy of GFP expressing HLE cells during experimental colonization of the chick lung at 7 days post-injection. Dissemination to the lung was measured as the number of metastatic nodules/colonies per lung. Data are expressed as the mean \pm SEM from three independent experiments. A total of 5 x105 HLE cells were grafted on the dropped CAM of 10-day-old chick embryos. Different doses of LY2109761 were administered on days three and five postinoculation of tumor cells. Tumors were then carefully excised from the individual 19 -day-old embryos and weighed. $* \mathrm{P}<0.05$, $* * \mathrm{P}<0.005, * * * \mathrm{P}<0.0005$ versus control. 
Fig. 1A.eps

A

HLE

HLF
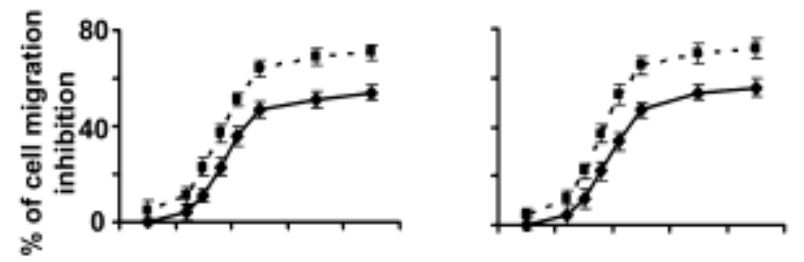

$\rightarrow-1$ add

$\cdots 2$ add

B
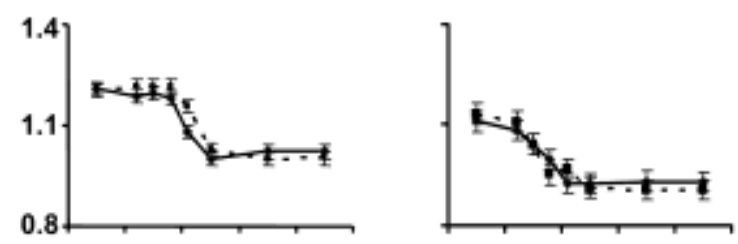

p-Smad-2

C
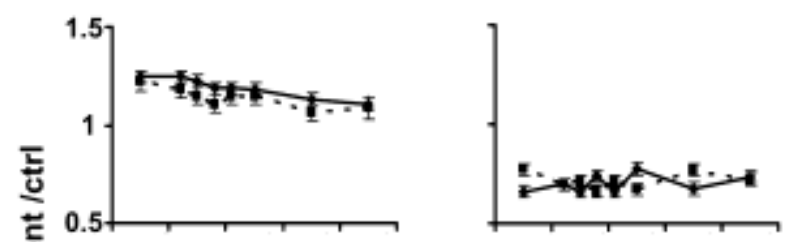

p-38-MAP-Kinase

D

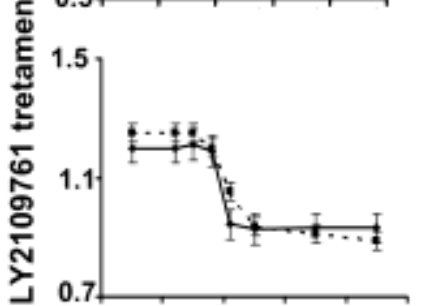

E
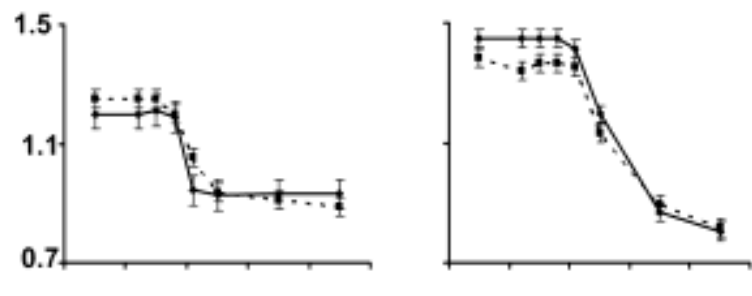

p-FAK

p- $\beta 1$-integrin

F
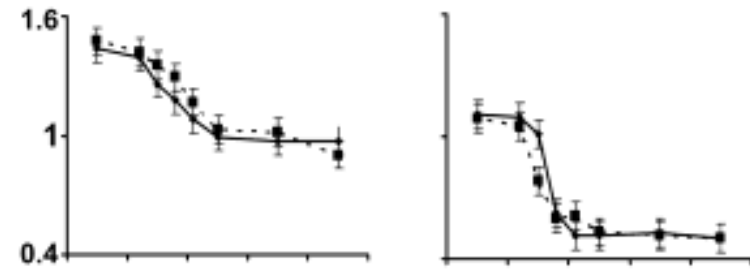

E-Cadherin

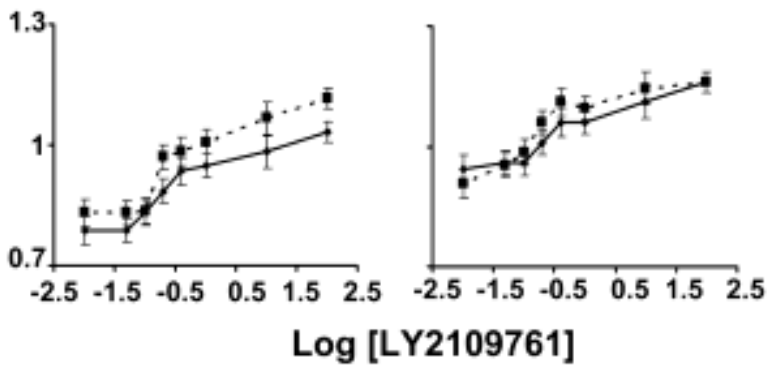


Fig.1B.eps

HLE
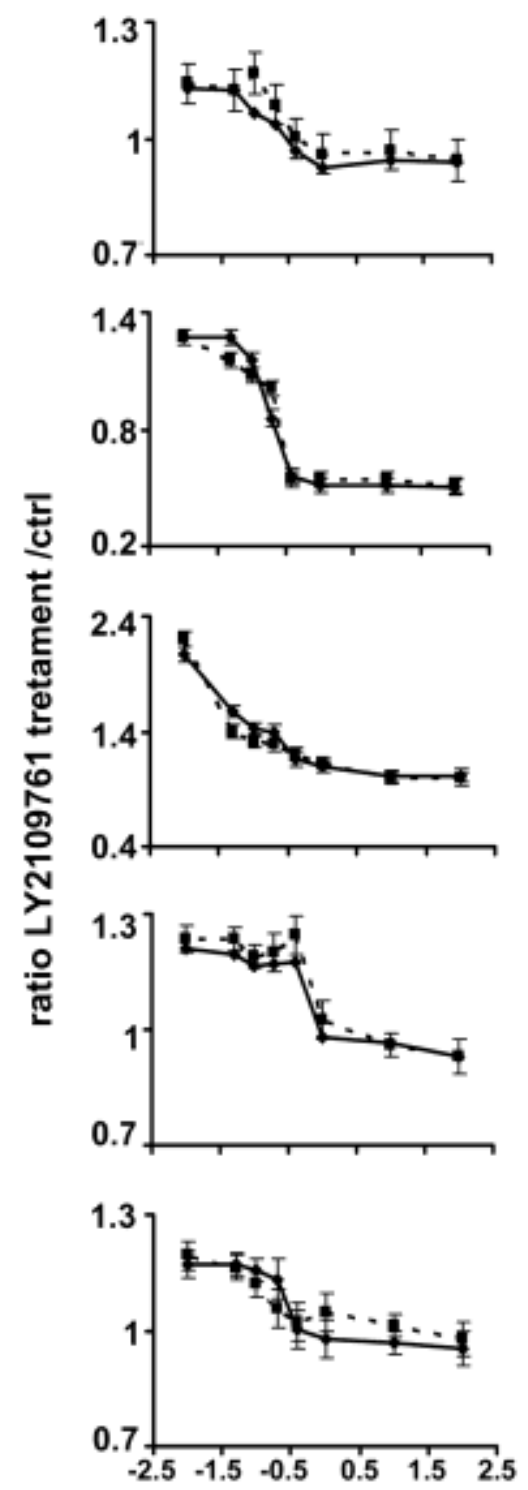

HLF

$\rightarrow 1$ add

$\cdots 2$ add
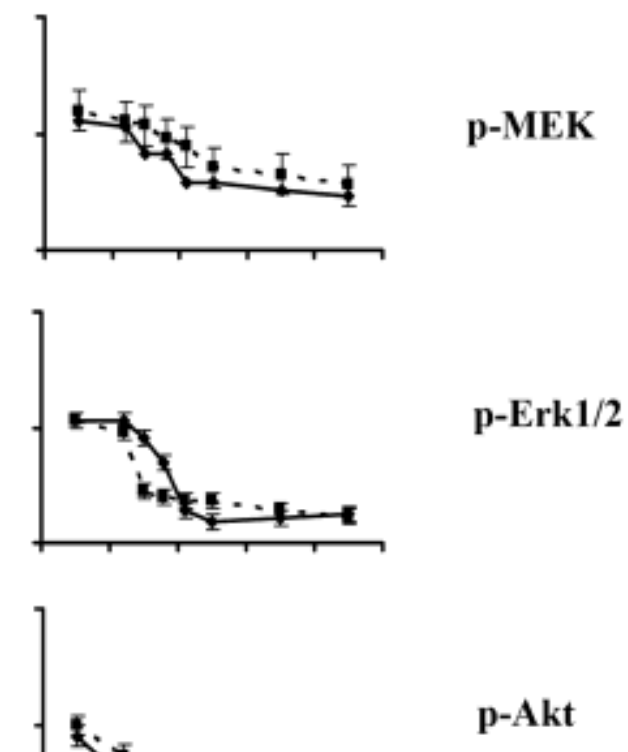

p-mTOR


Log [LY2109761] 
Fig 2.eps

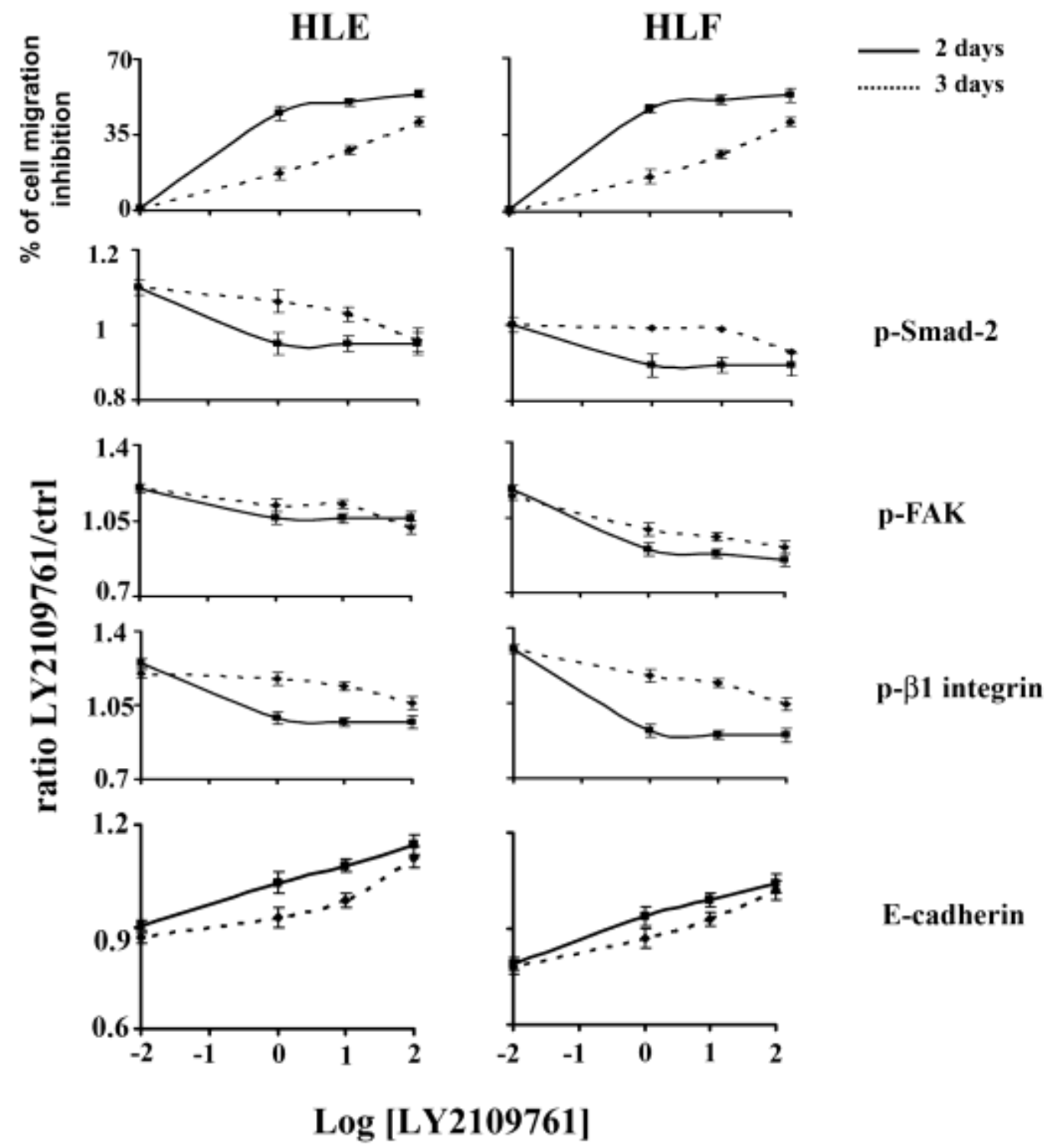


Fig.3.eps




Fig 4.eps

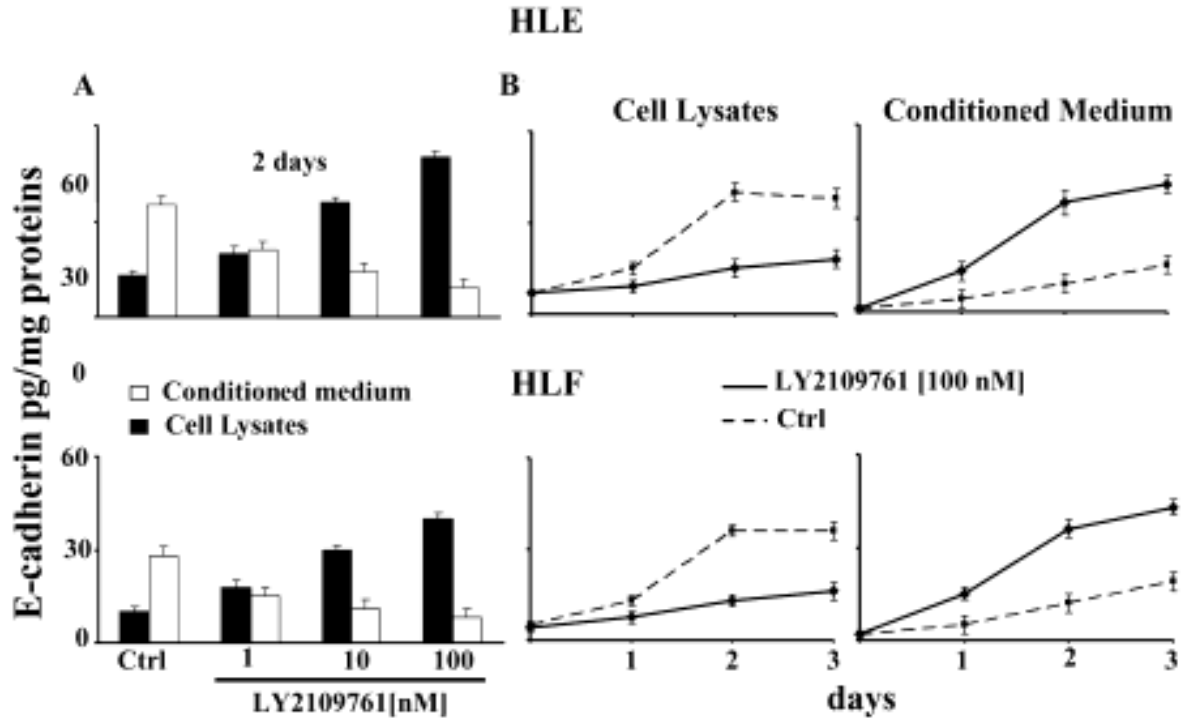


Fig 5.eps

A

HLE

HLF
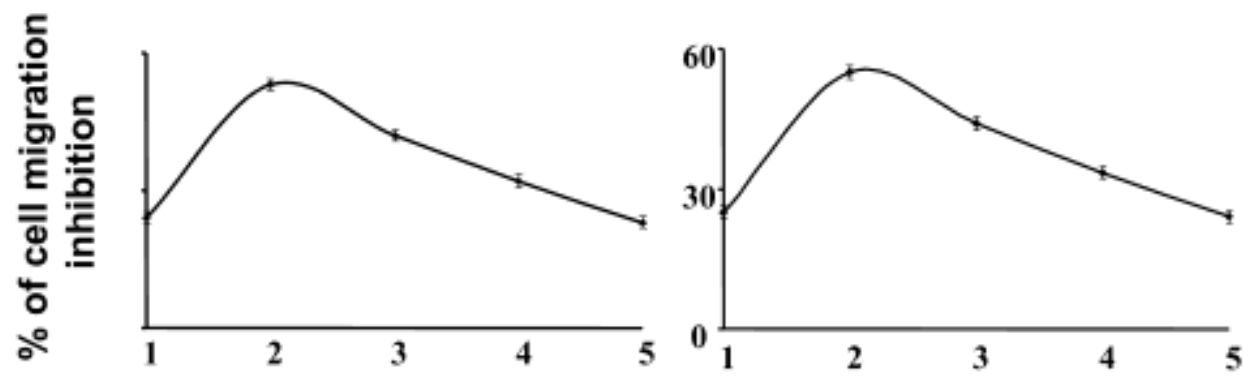

B

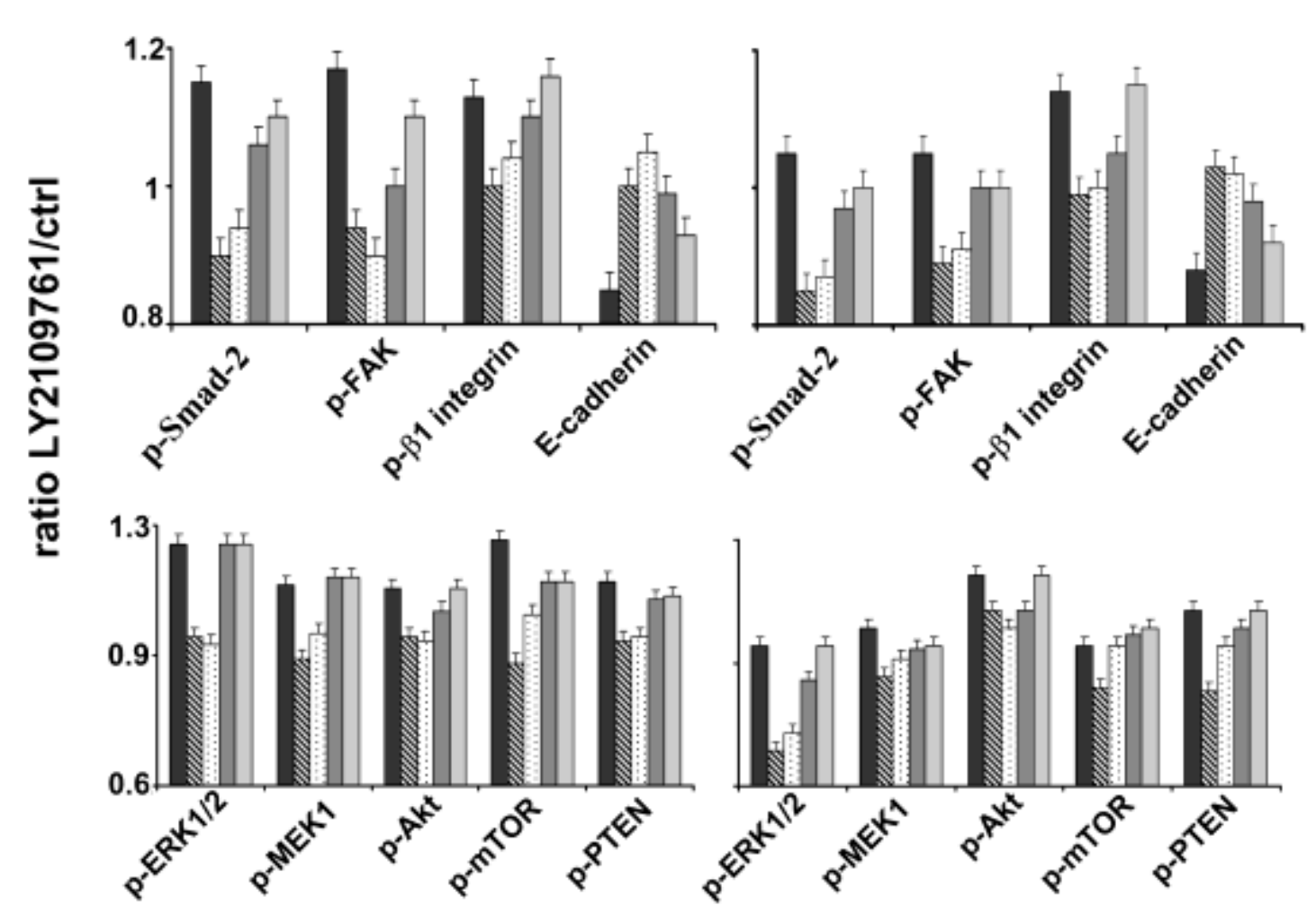

days
$\square 1$
$\mathbb{2} 2$
$\square 3$
$\square 4$
$\square 5$ 


\section{Fig 6.eps}

A

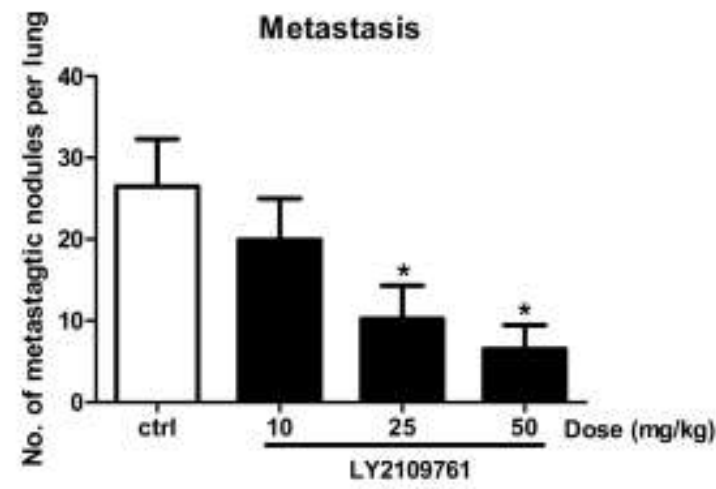

B

Inhibition of intravasation

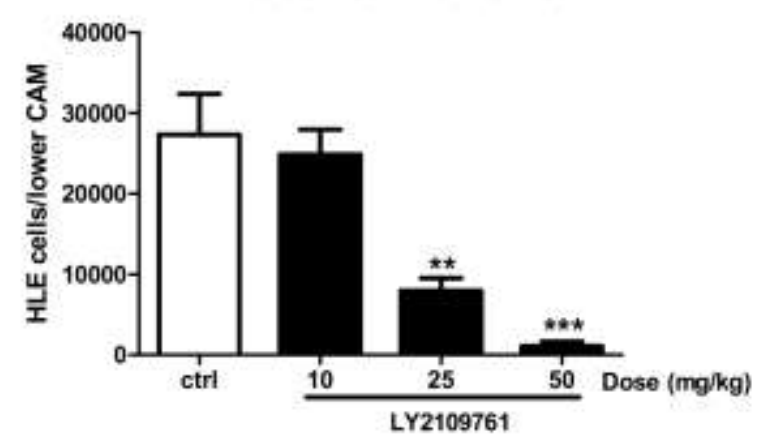

C Tumor Growth

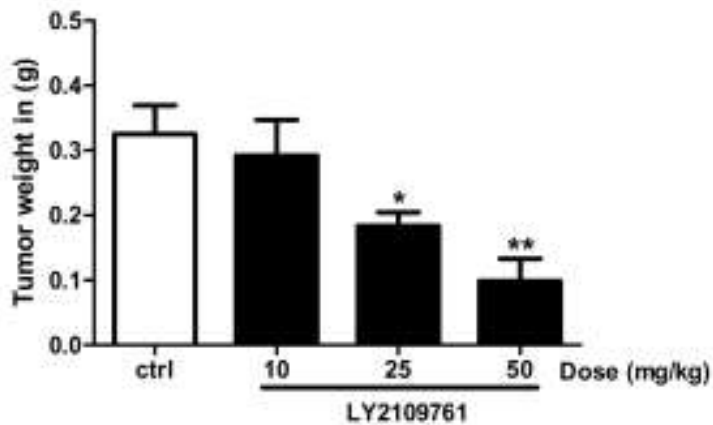

